\title{
The dependence of the Irregularity Parameter \\ Characterizing the Equatorial Ionospheric \\ Irregularities on the background Ionospheric Density
}

\section{Joshi, K. Groves, J. Retterer}

Journal of Nepal Physical Society

Volume 7, Issue 1, April 2021

(Special Issue: ANPA Conference, 2020)

ISSN: 2392-473X (Print), 2738-9537(Online)

\section{Editors:}

Dr. Santosh KC

San Jose State University, USA (Editor in Chief) Dr. Pashupati Dhakal

Thomas Jefferson National Accelerator Facility, USA Dr. Yadav Pandit

Baptist Health Science University, USA

Managing Editor:

Dr. Binod Adhikari

St. Xavier's College, Kathmandu, Nepal

JNPS, 7 (1), 1-5 (2021)

DOI: http://doi.org/10.3126/jnphyssoc.v7i1.36967

Published by:

Nepal Physical Society

P.O. Box: 2934

Tri-Chandra Campus

Kathmandu, Nepal

Email: npseditor@gmail.com

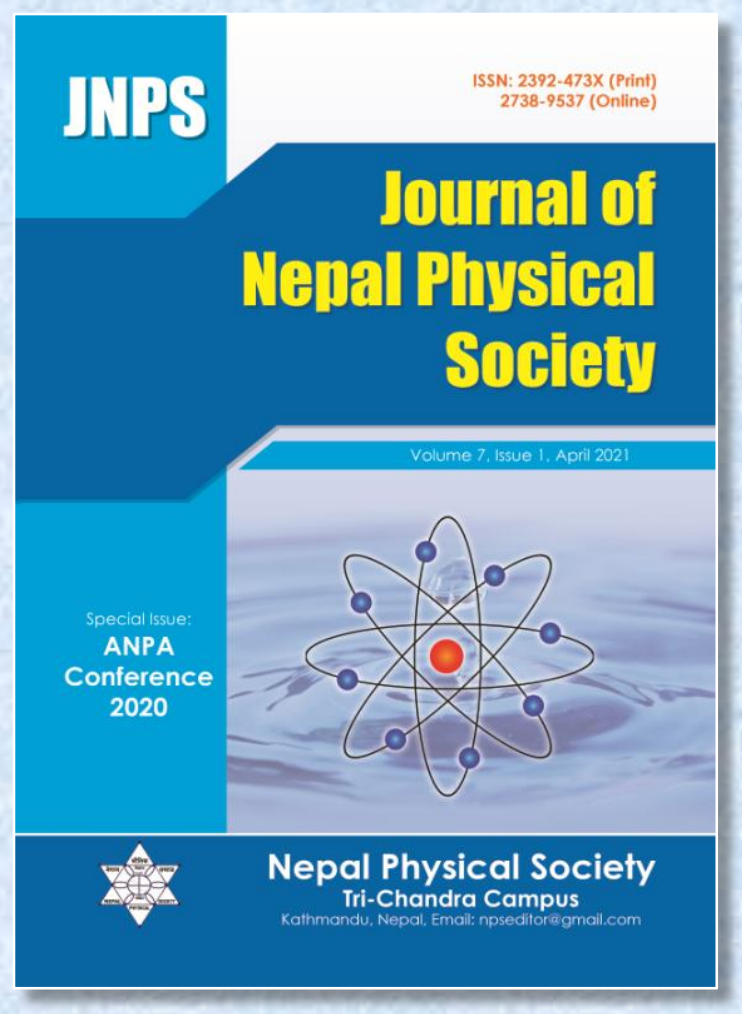




\title{
The dependence of the Irregularity Parameter Characterizing the Equatorial Ionospheric Irregularities on the Background Ionospheric Density
}

\author{
D. Joshi ${ }^{1}$, K. Groves ${ }^{2}$, J. Retterer ${ }^{2}$ \\ ${ }^{1}$ The University of Scranton, PA, USA \\ ${ }^{2}$ Institute for Scientific Research, Boston College, MA, USA \\ "Corresponding Email: devraj.joshi@ scranton.edu
}

Received: 7 August, 2020; Revised: 9 January, 2021; Accepted: 5 February, 2021

\begin{abstract}
The low-latitude ionosphere is characterized by large-scale instabilities in the post-sunset hours due to the distinct geometry of the earth's magnetic field lines at the equator. The magnetic field lines are horizontal at the equator contributing to the high vertical drift velocity of the plasma bubbles growing from the bottomside of the ionospheric F-region. The phenomenon, commonly known as equatorial spread $\mathrm{F}$, is an important problem in aeronomy as it can cause radio wave scintillation effects representing the most critical impacts of space weather on manmade technologies, such as satellite communications and global navigation satellite systems (GNSS). Here, we report results on the dependence of the peak heights of the irregularities at the magnetic equator, also called as apex-altitude, on solar flux by analyzing in-situ observations made on-board the Communications/Navigations Outage Forecasting System (C/NOFS) satellite mission. Our analysis indicates the median of the peak-height distributions of the irregularities increases linearly from about $491 \mathrm{~km}$ at solar minimum to $737 \mathrm{~km}$ during solar maximum. The Physics-Based Model (PBMOD) has been used to confirm the spacebased observational results and we find the field-line integrated conductivity is the key parameter which controls the peak-heights of the irregularities. In this investigation, we also seek to understand the possible dependence of the irregularity parameter characterizing the equatorial ionospheric irregularities on the background ionospheric density.
\end{abstract}

Keywords: Equatorial ionospheric irregularities, Physics-based model (PBMOD), Ionospheric density.

\section{INTRODUCTION}

The in-situ ion-density measurements made by the Planar Langmuir Probe (PLP) sensor on-board C/NOFS [1] satellite has been analyzed to understand the climatological features of the equatorial ionospheric irregularities (EIA) in several previous studies. Equatorial plasma irregularities are caused by the non-linear evolution of the generalized Rayleigh Taylor (RT) instability $[2,3]$ in which the bottomside low-density plasma drifts upward into the high-density plasma much like the rise of air bubbles in a liquid [4]. The ionospheric irregularities are generically called 'spread F' owing to the spread observed in the ionograms when they were first observed. Since their first discovery by Berkner and Wells [5] in 1934, these irregularities have been extensively studied in a variety of experiments involving sounding rockets [6], ground-based radars, satellites, in-situ probes, conventional ionosondes, topside ionosondes, airglow measurements and satellite beacons [7]. The irregularities have been an active field of research due to both academic interest and practical applications as these irregularities because radio wave scintillations disrupting satellite, communication, navigation, surveillance and aviation systems.

Following the important works by Haerendel [2] and Balsley et al. [8], the disturbances in the equatorial ionospheric region started to be 
understood as a flux-tube aligned interhemispheric effect explained by the gravitational RayleighTaylor (GRT) flux tube interchange instability. In this scheme, the high plasma density magnetic flux tubes at the bottom-side of the ionospheric F region are replaced by the lower plasma density flux tubes from below similar to the hydrodynamic Rayleigh Taylor instability. The magnetic field lines are horizontal at the equator contributing to the high vertical drift velocity, due to the polarization electric fields created by the disturbances in the plasma, of the plasma bubbles growing from the bottomside of the ionospheric F-region.

a)

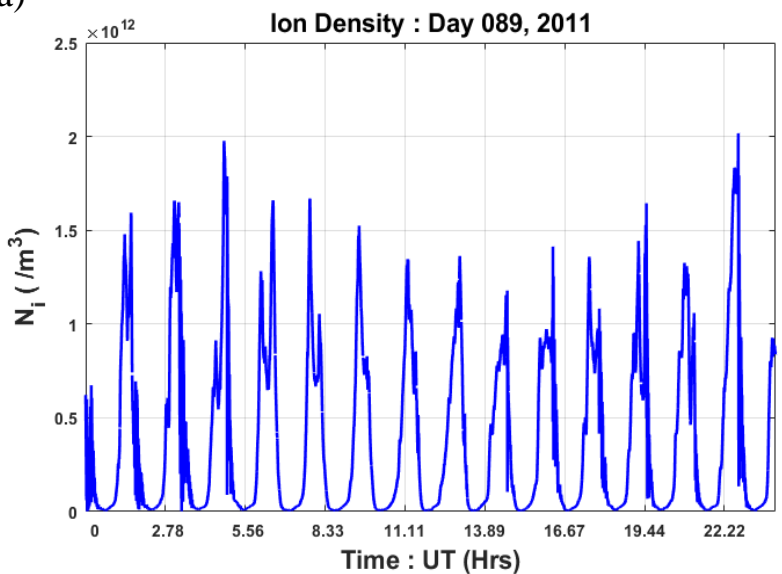

b)
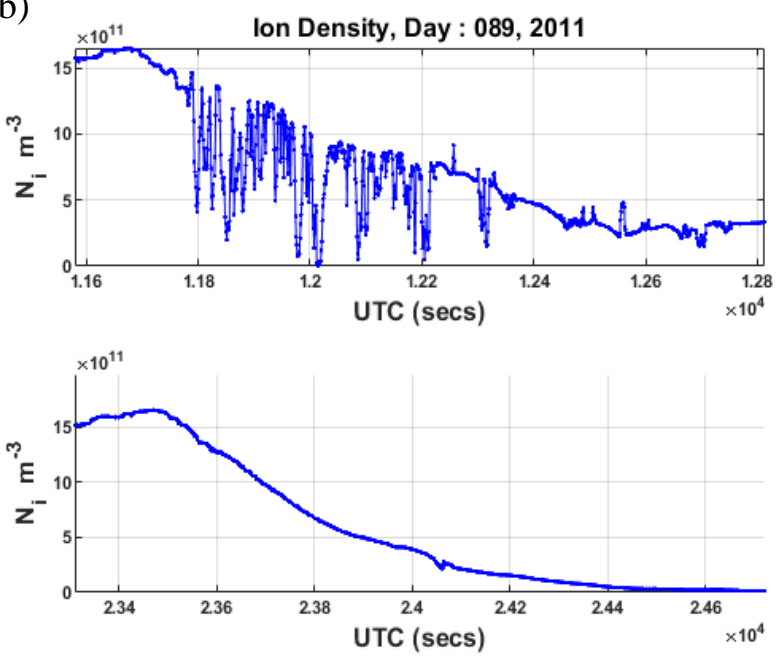

Fig. 1: (a) An example of ion-density measurements for UTC day 089, 2011 made by C/NOFS PLP sensor (above).

(b) lonospheric Irregularities in the upper subplot and smooth ion density measurements on the same day in the lower subplot (below).

In this work, we seek to characterize the spatial distribution of equatorial ionospheric irregularities (Fig. 1) and try to understand the dependence of the peak heights of the irregularities at the magnetic equator, also called as apex-altitude, on solar activity.
Since the irregularities map along the magnetic field lines, their height above the magnetic equator determines the spatial extent of the irregularities in latitude allowing us to identify regions affected by space weather impacts. In this investigation, we seek to understand the possible dependence of the irregularity parameter characterizing the equatorial ionospheric irregularities on the background ionospheric density. This is a critical examination of the irregularity parameter to confirm our results on the peak-height distributions of the irregularities at the magnetic equator and also several other studies which have employed similar definition of the irregularity parameter.

\section{OBSERVATIONS AND METHODOLOGY}

In our work, we use the following parameter $\sigma$ to identify ionospheric irregularities:

$$
\sigma(\%)=100 \times \frac{\left[\frac{1}{11} \sum_{i=1}^{11}\left(\log N_{\mathrm{i}}-\log N_{\mathrm{oi}}\right)^{2}\right]^{1 / 2}}{\left[\frac{1}{11} \sum_{i=1}^{11} \log N_{\mathrm{oi}}\right]}
$$

where $N_{i}$ and $N_{o i}$ are the ion density and the linearly fitted value at the $i^{\text {th }}$ data position. This definition has been used in previous studies aimed $[9,10]$ at investigating ionospheric irregularities. Equation (1) is the standard deviation of ion density variation in logarithmic scale for an 11-s segment of data. In our work, while applying $\sigma$ defined in equation (1) as the parameter to identify irregularities, we have taken the background density $\left(\mathrm{N}_{\mathrm{oi}}\right)$ to be moving median of 200 points/secs. In this work, we set $\sigma>1.2 \%$ as the criterion to identify the ionospheric irregularities with ion-density units taken to be in $\mathrm{m}^{-3}$.

While understanding the observational results, we also analyze Physics Based Model (PBMOD) ionospheric model results to determine if a physicsbased model can reproduce the observed dependence of bubble height on solar activity. PBMOD [11] ionospheric model, as described in the reference, is a system of Physics Based MODels that describes the three-dimensional timedependent evolution of the low-latitude ionosphere on several different spatial scales: globally it provides the plasma density and composition at altitudes between 90 and $2000 \mathrm{~km}$; at finer scales it describes the development of fluid plasma turbulence within this region and the resulting radio scintillation. We varied the seed perturbation in the model to compare with the C/NOFS observational results. The seed value which produced non-linear 
effects in the model results and closely matched with the C/NOFS observational results was chosen for further analysis so that we could look for physical parameters accessible through the model runs to understand the physical mechanism responsible for the peak-heights of the equatorial ionospheric irregularities at the magnetic equator.

\section{VARIATION OF PEAK APEX ALTITUDE DISTRIBUTIONS OF EIA WITH SOLAR ACTIVITY}

We now proceed with our objective of determining the apex-altitude distribution of the bubbles. We take data from 2008 through 2010 as low solar activity period. Since the first half of 2011 has transitional F10.7 values, we include data from the second half of 2011 through 2014 as high solar activity period. The sampling window for both solar low and high activity years includes active seasons Jan - April and Sep - Dec., Local-Time 20 $-24 \mathrm{LT}$ and longitudinal region : $80^{\circ} \mathrm{W}: 10^{\circ} \mathrm{E}$.

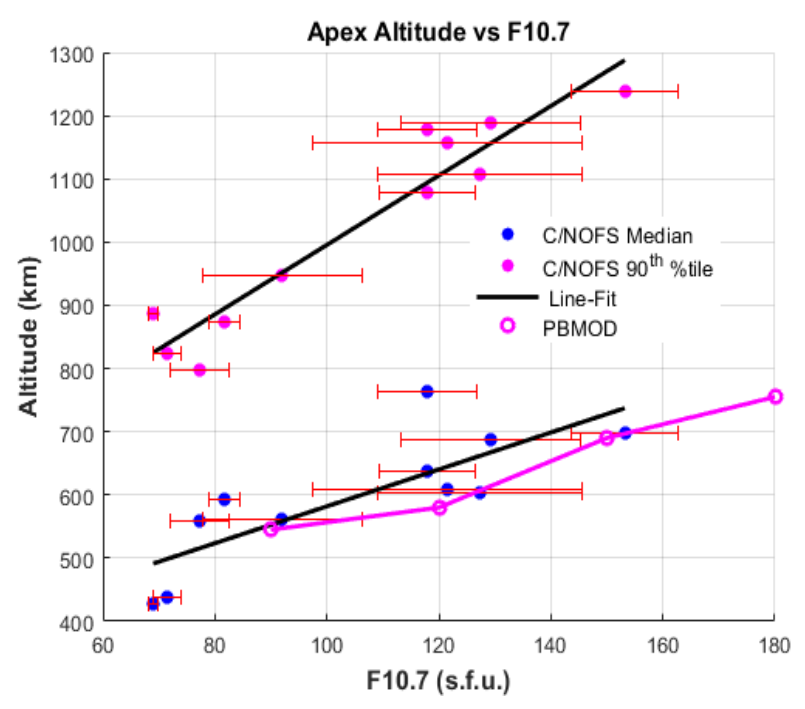

Figure 2: Apex Altitude vs F10.7 plot shows the evolution of the median peak apex-altitudes from low solar activity year 2008 to high solar activity year 2014 . The line fit shows that the irregularities rise from about $491 \mathrm{~km}$ at solar minimum to $737 \mathrm{~km}$ during solar maximum. The line fit has higher slope for the $90^{\text {th }}$ percentile peak apex-altitudes. The PBMOD results for the variation of the plasma bubble apex altitude with solar flux matches closely with that of the median values of the peak apex-altitude distributions deduced from C/NOFS observations.

We are interested in finding out the 'True Distribution' of peak-altitudes of the bubbles at the magnetic equator. It is the peak altitudes of the bubbles at the magnetic equator which determine their latitudinal extent as the irregularities map along the equipotential magnetic field lines in either side of the hemispheres. The True Distribution is estimated from the Observed Distribution based on the physical reality that the number of observed distribution at an apex altitude is integral of the true distribution at apex-altitudes equal and higher than that of the observed distribution. Conversely, all higher altitude bubbles at the magnetic equator pass through the lower altitudes beneath them. Mathematically,

$$
\mathrm{M}_{\mathrm{zo}}=\int_{z o}^{\infty} N(z) d z,---(3)
$$

where, $\mathrm{M}_{\mathrm{zo}}$ is observed distribution and $\mathrm{N}(\mathrm{z})$ is true distribution.

We apply this approach to deduce the peak apex altitude distributions from the observed apex altitude distributions of the equatorial ionospheric irregularities as observed by the sensor on-board C/NOFS satellite. The variation of the peak apexaltitude distributions of the equatorial ionospheric irregularities with solar activity is as seen in Fig. 2. The median of the distributions varies from $491 \mathrm{~km}$ at solar minimum years to $737 \mathrm{~km}$ at solar maximum years.

\section{INFLUENCE OF THE BACKGROUND DENSITY ON THE IRREGULARITY PARAMETER}

We seek to understand the possible influence of the background ionospheric density on the irregularity parameter characterizing the equatorial ionospheric irregularities. This is a critical examination of the parameter to confirm our results on the peak-height distributions of the irregularities at the magnetic equator and also other studies which have employed similar definition of the irregularity parameter. The scatter plots for the sigma, absolute deviation in linear scale and the background density in the linear scale with respect to the physical altitude all have a 'steeper' slope. The sigma values closer to the magnetic equator (low apex altitude) at lower physical altitudes are higher (Fig. 3) than those farther from the magnetic equator (high apex altitude). But this does not hold true for the irregularity parameter defined in terms of the linear scale (absolute deviation ratio). Hence, it requires more investigation to understand if the background density influences the irregularity parameters. 


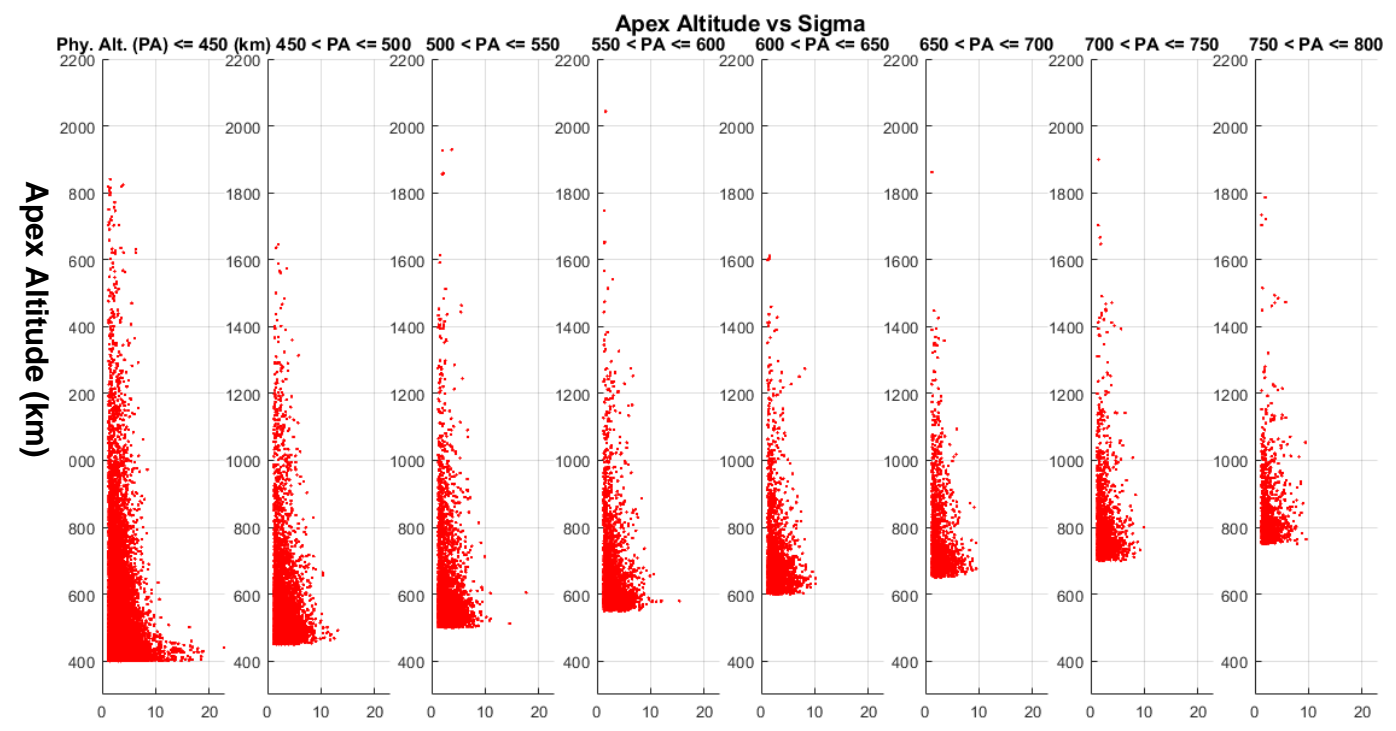

Sigma $(\sigma)$

Fig. 3: The scatter plot (bottom right) of the Sigma shows that sigma decreases in value as we go higher in physical and apex altitudes. This holds true for other irregularity parameters as well. The plot binned in physical altitude (top) shows sigma values closer to the magnetic equator are higher than those farther from the magnetic equator.

In the scatter plot, the physical altitude spans (400 - 850) km owing to the altitudinal range of the elliptical orbit of C/NOFS satellite.

In the scatter plot, the physical altitude spans $\sim(400-$ 850) $\mathrm{km}$ owing to the altitudinal range of the elliptical orbit of C/NOFS satellite. The red dots corresponding to the physical-altitudes in the image 'hide' the blue dots corresponding to the apex-altitudes. Hence, the apex-altitudinal range corresponds to $\sim(400-2200)$ $\mathrm{km}$ as the apex-altitude for a physical latitude is determined by the magnetic-field mapping between the geographic (physical) latitude and the geomagnetic equator. The higher values of Sigma at the low physical altitude and very close to the magnetic equator $(<500 \mathrm{~km})$ are possibly due to the strength of the irregularities as they are just developed into non-linear stage which would fade in the strength as they grow upwards. More investigation will be required to test this hypothesis which will constitute future work on this investigation.

\section{CONCLUSIONS}

The research results presented in this paper make

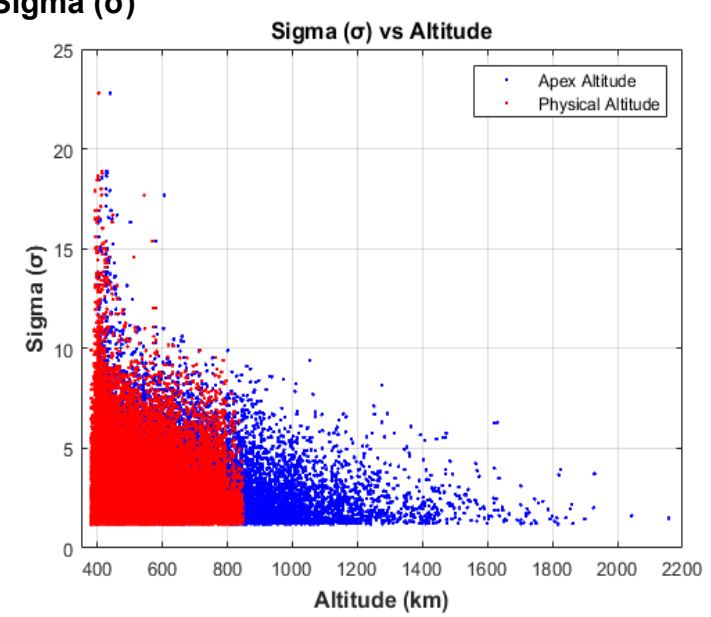

an investigation into altitude distributions of equatorial ionospheric irregularities at the magnetic equator. We studied the apex-altitude distributions of equatorial ionospheric irregularities for low and high solar activity years. We used PBMOD (Physics Based $M O D$ els) to see if model predicts similar effects of solar activity on the altitude of ionospheric irregularities at magnetic equator as of C/NOFS observations [12]. We summarize our investigation results as following:

1. Our analysis indicates that the median height distribution of bubbles increases linearly from about $491 \mathrm{~km}$ at solar minimum to $737 \mathrm{~km}$ during solar maximum. These results apply to the $-80^{\circ} \mathrm{W}$ to $10^{\circ} \mathrm{E}$ longitude sector. Other longitude sectors may have different altitude distributions. Our occurrence statistics suggest that bubbles in this longitude sectors are higher than in other regions on average. 
2. A physics-based model was used to confirm our findings. In the model it appears that field-line integrated conductivity is the key determinant of terminal bubble altitude. Specifically, when the field-line integrated conductivity inside the bubble is equal to that of the background ionosphere the polarization electric field that propels the bubble upwards vanishes and the bubble ceases to rise further.

3. These results are the first observational confirmation of bubble altitude as a function of solar flux and are valuable to the development of improved scintillation mapping models for both real-time and post-processing applications.

4. More investigation is required to reach towards unambiguous conclusions on the influence of background density on the irregularity parameter characterizing the ionospheric irregularities.

\section{ACKNOWLEDGEMENTS}

We would like to acknowledge Dr. Patrick Roddy, Dr. Chaosong Huang at Air Force Research Laboratory, Albuquerque, New Mexico for helpful discussion towards completion of this work.

\section{EDITOR'S NOTE}

This manuscript was submitted to Association of Nepali Physicists in America (ANPA) Conference 2020 for publication in the special issue of Journal of Nepal Physical Society.

\section{REFERENCES}

[1] https://directory.eoportal.org/web/eoportal/ satellite-missions/c-missions/cnofs.

[2] Haerendel, G. Theory of equatorial spread F, technical report, Max Planck Inst. fur Extraterr. Phys. Munich, Germany (1973).

[3] Gentile, L. C.; Burke, W. J. and Rich, F. J. A climatology of equatorial plasma bubbles from DMSP 1989- 2004, Radio Sci., 41, RS5S21 (2006).

[4] Kil, H.; Paxton, L. J. and Oh, S. J. Global bubble distribution seen from ROCSAT-1 and its association with the evening prereversal enhancement, J. Geophys. Res., 114, A06307 (2009).

[5] Rodrigues, F. S.; Kelley, M. C.; Roddy, P. A.; Hunton, D. E.; Pfaff, R. F.; de La Beaujardière, O. and Bust, G. S. C/NOFS observations of intermediate and transitional scale-size equatorial spread $F$ irregularities, Geophys. Res. Lett., 36, L00C05 (2009).
[6] Joshi, D.; Groves, K. M.; McNeil, W.; Carrano, C.; Caton, R. G.; Parris, R. T.; Pederson, T. R.; Cannon, P. S.; Angling, M. and Jackson-Booth, N. HF propagation results from the Metal Oxide Space Cloud (MOSC) experiment. Radio Science, 52(6): 710-722 (2017).

[7] Thampi, S. V.; Yamamoto, M.; Tsunoda, R. T.; Otsuka, Y.; Tsugawa, T.; Uemoto, J. and Ishii, M. First observations of large-scale wave structure and equatorial spread F using CERTO radio beacon on the C/NOFS satellite, Geophys. Res. Lett., 36, L18111 (2009).

[8] Balsley, B. B.; Haerendel, G. and Greenwald, R. A. Equatorial spread $F$ : Recent observations and a new interpretation, $J$ Geophys. Res., 77(28): 5625-5628 (1972).

[9] Kil, H. and Heelis, R. A. Global distribution of density irregularities in the equatorial ionosphere, J. Geophys. Res., 103(A1): 407-417 (1998).

[10] Su, S. Y.; Liu, C. H.; Ho, H. H. and Chao, C. K. Distribution characteristics of topside ionospheric density irregularities: Equatorial versus midlatitude regions, J. Geophys. Res., 111, A06305 (2006).

[11] https://ccmc.gsfc.nasa.gov/RoR_WWW/pbmodrt/PBMOD-Text.html.

[12] Artificial and Natural Disturbances in the Equatorial Ionosphere: Results from the MOSC Experiment and the C/NOFS Satellite Mission (https://dlib.bc.edu/islandora/object/bcir\%3A108706). 